MATEC Web of Conferences 1, 10010 (2012)

DOI: $10.1051 /$ matecconf $/ 20120110010$

(C) Owned by the authors, published by EDP Sciences, 2012

\title{
Effects of in-plane inertia on natural frequency and stresses of long circular cylindrical shell
}

\author{
M. Rougui ${ }^{1, a}$ and A. Karimin ${ }^{2}$ \\ 1 Laboratory of Energy, Materials and Environment, High School of Technology, University Mohammed V Agdal, Av \\ Prince Heritor, Po box 227, Sale Morocco \\ 2 Laboratory of Mechanics, Faculty of Sciences Aïn Chock, PB 5366 Maârif, Casablanca, Morocco
}

\begin{abstract}
In this paper an analytical procedure is given to study the free-vibration characteristics of thin long circular cylindrical shells with simply supported boundary conditions. An approach based on Sander's nonlinear theory and Lagrange equations is employed. Influence of in-plane inertia on natural frequencies and stress for several modes shape is discussed. Natural frequencies for a number of particular cases are evaluated and compared with some available experimental and other analytical results in the literature. The distributions of the bending and axial stresses associated with the nonlinear modes shape are given and compared with those obtained via the linear theory.
\end{abstract}

\section{Introduction}

The study of the dynamic behavior of isotropic and orthotropic thin shells within the scope of linear and nonlinear models has been the subject of numerous works. Readers interested by these works can turn to [1] and [2] or to the review paper presented recently by [3], and to a more recent review paper by [4], in which a set of papers are discussed and commented. It can be observed that although several works exist in literature on nonlinear vibrations of shells, only few papers are available which are concerned with the nonlinear free vibration of shells of infinite length. Also, influence of inertia terms on nonlinear natural frequencies and their contribution on linear and nonlinear stress have been examined very rarely. However, many researchers have used certain simplifications and derived much simpler characteristic equations. The two widely used simplifications cited in literature are those made by [5] and [6] in which they neglected the tangential inertia terms of a cylindrical shell. [5] showed that for a cylindrical shell in which the circumferential wavelength is much less than the axial one, the characteristic equation is similar to that of a lateral vibrating Euler-Bernoulli beam. Applying his simplification to the Donnell theory along with the neglect of tangential inertia, [5] was able to come up with some good results. Such a procedure was similarly carried out by [7]. As mentioned above, a further investigation of the neglect of tangential inertia and its effects on theoretical results was carried out by [6]. The main aim of the present paper is to investigate the effects of in-pane inertia (circumferential and longitudinal) on natural frequencies and stresses of long circular cylindrical shell.

\section{Mathematical modelling}

Consider an isotropic, elastic, circular cylindrical shell of uniform thickness $h$, length $L$, radius $R$, Young's modulus

a e-mail: rougui93@yahoo.fr
$E$ and Poisson's ratio $v$.

The elastic strain energy $V$ of a circular cylindrical shell, under Love's hypothesis $\sigma_{z z}=\sigma_{z x}=\sigma_{x y}=0$, given by

$$
V=\frac{1}{2} \int_{-\frac{h}{2}}^{\frac{h}{2}} \int_{0}^{2 \pi R} \int_{0}^{L}\left(\sigma_{x x} \epsilon_{x x}+\sigma_{y y} \epsilon_{y y}+\tau_{x y} \gamma_{x y}\right) d z d x d y
$$

where $\sigma_{x x}, \sigma_{y y}$ and $\tau_{x y}$ are Kirchhoff stresses and $\epsilon_{x x}, \epsilon_{y y}$ and $\gamma_{x y}$ are Green's strains. The simplicity of Eq. (1) is due to the Lagrangian description of the shell, which allows integration over the shell in the original undeformed configuration. The Kirchhoff stresses for a homogeneous and isotropic material ( $\sigma_{z z}=0$ case of plane stress) are given by

$$
\begin{aligned}
\sigma_{x x}=\frac{E}{1-v^{2}}\left(\epsilon_{x x}+v \epsilon_{y y}\right), \sigma_{y y} & =\frac{E}{1-v^{2}}\left(\epsilon_{y y}+v \epsilon_{x x}\right) \\
\tau_{x y} & =\frac{E}{2(1+v)} \gamma_{x y}
\end{aligned}
$$

The strain components $\epsilon_{x x}, \epsilon_{y y}$ and $\epsilon_{x y}$ at an arbitrary point of the shell are related to the middle surface strains $\epsilon_{x, 0}, \epsilon_{y, 0}$ and $\epsilon_{x y, 0}$ and to the changes in the curvature and torsion of the middle surface $k_{x}, k_{y}$ and $k_{x y}$ by the following three relations

$$
\begin{gathered}
\epsilon_{x x}=\epsilon_{x, 0}+z k_{x} \\
\epsilon_{y y}=\epsilon_{y, 0}+z k_{y} \\
\gamma_{x y}=\gamma_{x y, 0}+z k_{x y}
\end{gathered}
$$

where $z$ is, as usual, the distance of the arbitrary point of the shell from the middle surface.

The expressions $\epsilon_{x, 0}, \epsilon_{y, 0}, \gamma_{x y, 0}, k_{x}, k_{y}$ and $k_{x y}$ are given by nonlinear Sanders shell theory. The boundary conditions and kinetic energy are given by:

$$
\begin{gathered}
N_{x}=M_{x}=v=w=0 \text { at } x=0, x=L \\
T=\frac{\rho h}{2} \int_{0}^{L} \int_{0}^{2 \pi R}\left[\left(\frac{\partial u}{\partial t}\right)^{2}+\left(\frac{\partial v}{\partial t}\right)^{2}+\left(\frac{\partial w}{\partial t}\right)^{2}\right] d x d y
\end{gathered}
$$


where $u, v$ and $w$ are the displacements of a generic point in the longitudinal, circumferential and radial direction, respectively; $N_{x}$ is the normal force, and $M_{x}$ is the bending moment per unit length.

The expressions of $u, v$ and $w$ which satisfying all the boundary conditions can be presented in this form

$$
\begin{aligned}
& u(x, y, t)=q_{1}(t) \cos \left(\frac{m \pi x}{L}\right) \cos \left(\frac{n y}{R}\right) \\
& v(x, y, t)=q_{2}(t) \sin \left(\frac{m \pi x}{L}\right) \sin \left(\frac{n y}{R}\right) \\
& w(x, y, t)=q_{3}(t) \sin \left(\frac{m \pi x}{L}\right) \cos \left(\frac{n y}{R}\right)
\end{aligned}
$$

where $q_{1}(t), q_{2}(t)$ and $q_{3}(t)$ are generalized coordinates. $m$ and $n$ are the axial halfwaves and circumferential waves numbers, respectively.

Lagrange's equations for a system of 3 degrees of freedom, which leads to:

$$
\frac{d}{d t}\left(\frac{\partial L}{\partial \dot{q}_{i}}\right)-\frac{\partial L}{\partial q_{i}}=0
$$

with $L=T-V$.

Replacing in this equation $\mathrm{T}$ and $\mathrm{V}$ by their expressions given above leads to the following set of nonlinear differential equations:

$$
\begin{aligned}
q_{1}(t) & +\lambda_{1} q_{1}(t)+\Gamma_{l, u}\left(q_{2}(t), q_{3}(t)\right) \\
& +\Gamma_{N l, u}\left(q_{1}(t), q_{2}(t), q_{3}(t)\right)=0 \\
\ddot{q_{2}}(t) & +\lambda_{2} q_{2}(t)+\Gamma_{l, v}\left(q_{1}(t), q_{3}(t)\right) \\
& +\Gamma_{N l, v}\left(q_{1}(t), q_{2}(t), q_{3}(t)\right)=0 \\
\ddot{q_{3}}(t) & +\lambda_{3} q_{3}(t)+\Gamma_{l, w}\left(q_{1}(t), q_{2}(t)\right) \\
& +\Gamma_{N l, w}\left(q_{1}(t), q_{2}(t), q_{3}(t)\right)=0
\end{aligned}
$$

where $\Gamma_{l}$ and $\Gamma_{N l}$ denote linear and nonlinear operators in the set of equations.

\subsection{Natural frequency}

After neglecting nonlinear terms of Eqs. (12-14) and assume harmonic balance hypothesis

$$
q_{1}(t)=A \cos (\omega t), q_{2}(t)=B \cos (\omega t), q_{3}(t)=C \cos (\omega t)
$$

the following set of equations is transformed to the matrix form given by

$$
M\left(\omega_{m, n}\right) \Delta=0
$$

where $A, B$ and $C$ are the contribution coefficients and $\Delta=(A, B, C)^{T}$.

In this work we treat two cases, (a): with longitudinal inertia $(\ddot{i})$ and (b): without circumferential inertia (ï).

Solving the system of obtained equations was made for the same mechanical and geometrical configuration of the long circular cylindrical shell treated experimentally in [8].

Three natural frequencies are obtained and associated to the radial, axial and circumferential mode shapes.Usually, the lowest frequency is associated with a motion that is primarily radial.

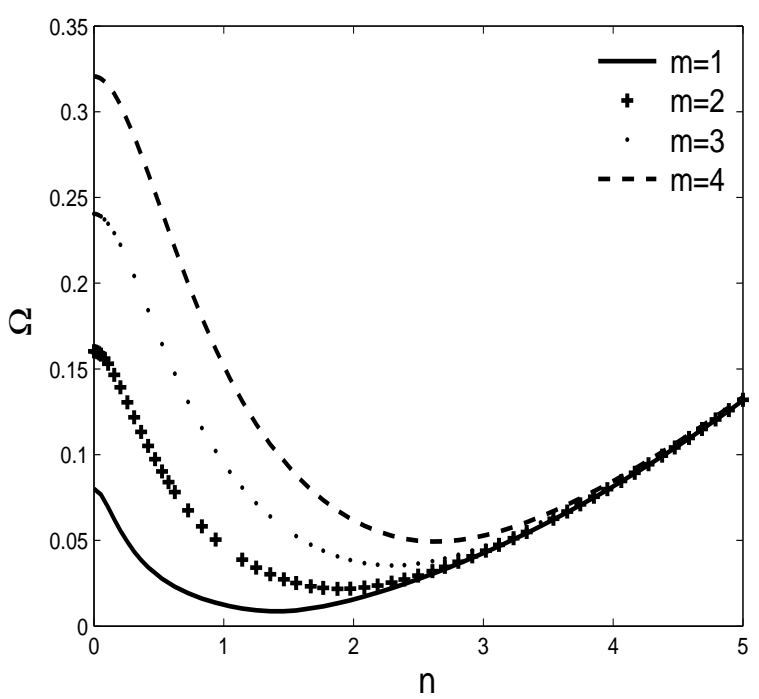

Fig. 1. Variation of non-dimensional frequency parameter $\Omega$ with $\mathrm{n} ; L / R=22.67, R / h=51.72, v=0.33$.

Table 1. Comparison between theoretical and experimental results of natural frequencies $(\mathrm{Hz})$ for different mode shapes in the case (a): with in-plane inertia; $L / R=22.67, R / h=51.72$.

\begin{tabular}{lllll}
\hline $\mathrm{m}$ & $\mathrm{n}$ & present work $^{a}$ & Experiment $^{[8]}$ & error $^{a}(\%)$ \\
1 & 0 & 901.07 & 842.5 & 6.95 \\
1 & 1 & 140.15 & 138.4 & 1.26 \\
1 & 2 & 175.26 & 190.3 & 7.90 \\
1 & 3 & 478.39 & 502.2 & 4.74 \\
1 & 4 & 915.68 & 884.4 & 3.54 \\
2 & 1 & 522.73 & 464.7 & 12.49 \\
2 & 3 & 488.18 & 477 & 2.34 \\
2 & 4 & 920.04 & 887 & 3.73 \\
3 & 2 & 427.76 & 496.6 & 13.86 \\
3 & 3 & 521.01 & 558.9 & 6.78 \\
3 & 4 & 930.47 & 981.6 & 5.21 \\
4 & 2 & 694.10 & 679.8 & 2.10 \\
4 & 4 & 951.24 & 945.8 & 0.58 \\
\hline
\end{tabular}

The results obtained in this work for the two cases considered are presented in Tabs. 1 and 2. In Fig. 1 we represent the non-dimensional frequency $\Omega=\frac{\rho\left(1-v^{2}\right) R^{2}}{E} \omega$.

Analysis of the obtained results shows that they are in good agreement if one takes into account the in-plane inertia. If applicable, the calculation error exceeds $60 \%$ which justifies well the effect of in-plane inertia of the behavior of the shell.

\subsection{Stress}

In this section we study the stresses with the two cases previously cited. The results obtained are shown in Figs. 2 and 3. In Fig. 2, for both longitudinal positions $L$ and $L / 2$ the axial linear and nonlinear stress with and without in-plane inertia vary in opposite directions from a radial value of the contribution less than $h$.

In Fig. 3, for both longitudinal positions $L$ and $L / 2$ the circumferential linear and nonlinear stress with and without in-plane inertia vary in the same direction with radial value of the contribution. In Figs. 2 and 3 the circumferential and 

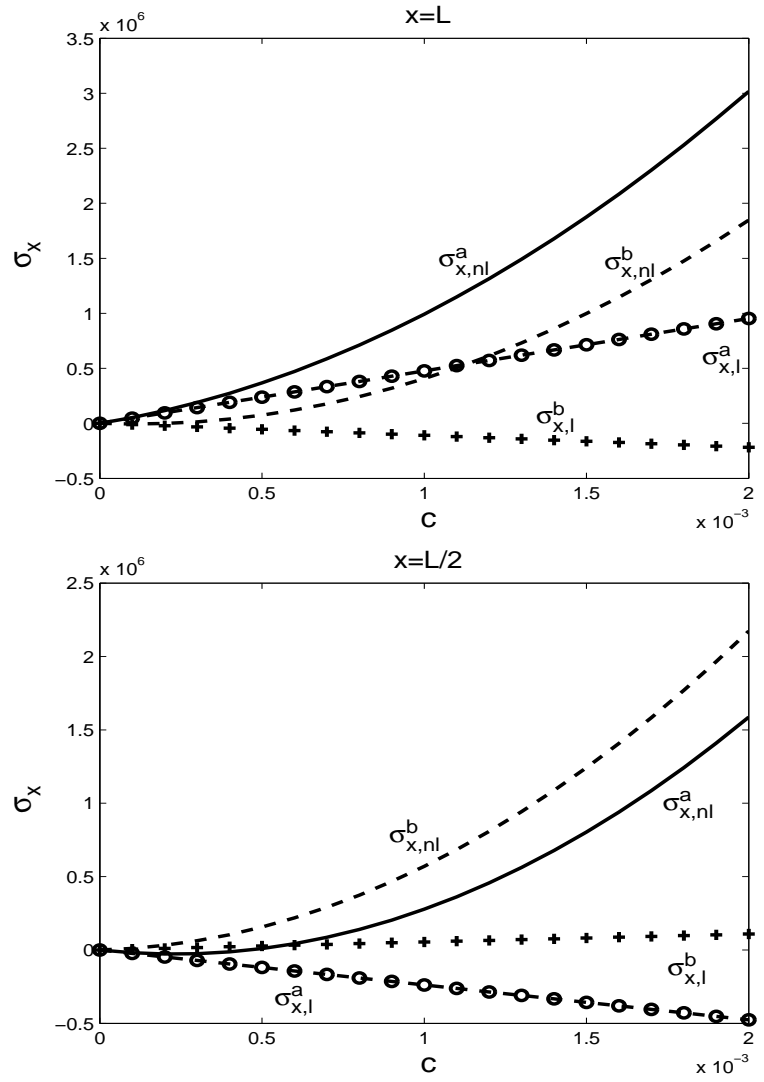

Fig. 2. The axial stress $\sigma_{x}$ versus transverse response for $m=$ $2, n=3$ in $y=2 \pi R$ and two positions for $x$.
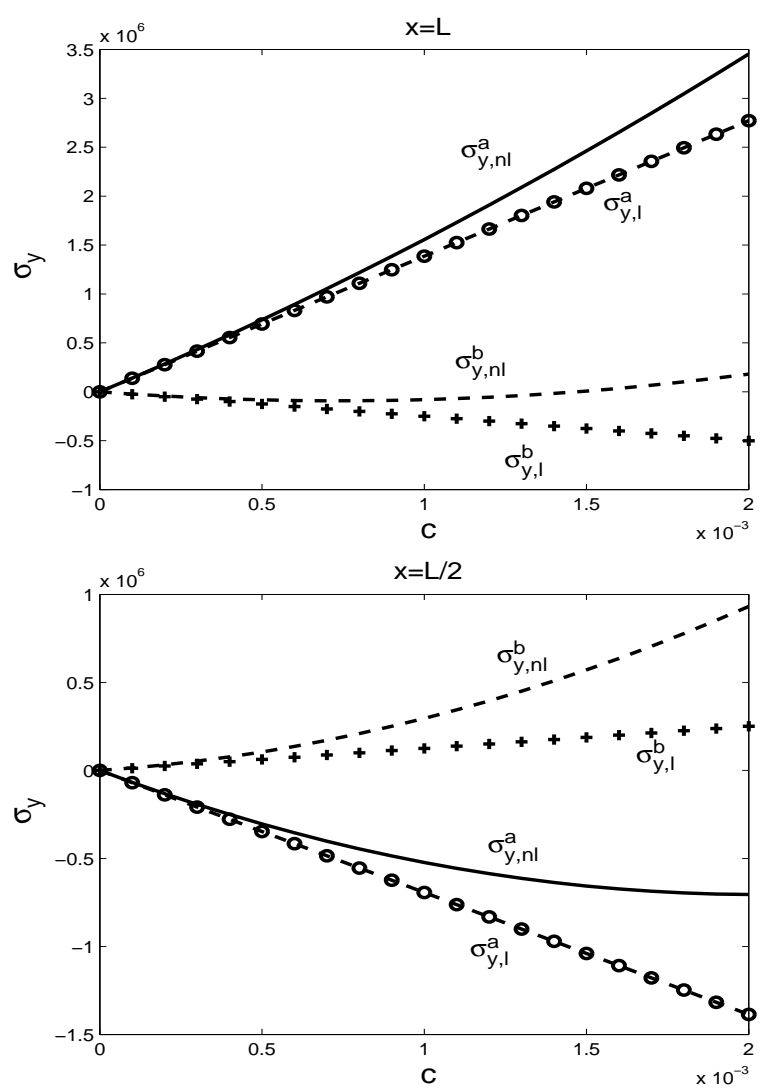

Fig. 3. The circumferential stress $\sigma_{y}$ versus transverse response for $m=2, n=3$ in $y=2 \pi R$ and two positions for $x$.
Table 2. Comparison between theoretical and experimental results of natural frequencies $(\mathrm{Hz})$ for different mode shapes in the case (b): without in-plane inertia; $L / R=22.67, R / h=51.72$.

\begin{tabular}{cclll}
\hline $\mathrm{m}$ & $\mathrm{n}$ & ${\text { present } \text { work }^{b}}^{b}$ & Experiment $^{[8]}$ & error $^{b}(\%)$ \\
1 & 0 & 943.95 & 842.5 & 12.04 \\
1 & 1 & 199.64 & 138.4 & 44.25 \\
1 & 2 & 196.10 & 190.3 & 3.05 \\
1 & 3 & 504.37 & 502.2 & 0.43 \\
1 & 4 & 943.95 & 884.4 & 6.73 \\
2 & 1 & 755.91 & 464.7 & 62.67 \\
2 & 3 & 514.96 & 477 & 7.96 \\
2 & 4 & 948.61 & 887 & 6.95 \\
3 & 2 & 481.06 & 496.6 & 3.13 \\
3 & 3 & 550.01 & 558.9 & 1.59 \\
3 & 4 & 959.63 & 981.6 & 2.24 \\
4 & 2 & 782.95 & 679.8 & 15.17 \\
4 & 4 & 981.40 & 945.8 & 3.76 \\
\hline
\end{tabular}

axial stress with in-plane inertia are larger than those without in-plane inertia for $x=L$; for the second position, we have the opposite behavior.

\section{Conclusion}

Results obtained in this paper are given in the form of table and figures. A good agreement is obtained between the corresponding experimental and analytical results available recently in the literature. The present result confirms the importance of the in-plane inertia terms and their influence on the behavior of linear and non linear thin shells.

\section{References}

1. W. Leissa, Vibration of Shells (US Government Printing Office, Washington DC 1973) 288.

2. D. A. Evensen, AIAA Journal 6, (1968) 1401-1403.

3. M. Amabili, M. P. Paidoussis, Applied Mechanics Reviews 56, (2003) 349-381.

4. F. Moussaoui, G. Benamar, Journal of Sound and Vibration 255, (2002) 161-184.

5. Y. Y. Yu, Journal of Applied Mechanics 22, (1955) 547552.

6. K. Forsberg, National Aeronautics and Space Administration NASA CR-613, (1966) 547-552.

7. V. I. Weingarten, Journal of the American Institute of Aeronautics and Astronautics 2, (1964) 717-722.

8. A. Farshidianfar, M. H. Farshidianfar, M. J. Crocker, W. O. Smith, Journal of Sound and Vibration 330, (2011) 3381-3399. 